\title{
The novel dual PI3K/mTOR inhibitor GDC-0941 synergizes with the MEK inhibitor U0126 in non-small cell lung cancer cells
}

\author{
ZU-QUAN ZOU ${ }^{1 *}$, LI-NA ZHANG $^{1 *}$, FENG WANG $^{2}$, JÉRÔME BELLENGER ${ }^{3}$, \\ YIN-ZHUO SHEN $^{1}$ and XIAO-HONG ZHANG ${ }^{1}$ \\ ${ }^{1}$ Medical School, Ningbo University; ${ }^{2}$ Clinical Laboratory, Lihuili Hospital, Ningbo 315211, P.R. China; \\ ${ }^{3}$ UMR 866 Physiopathologie des Dyslipidémies, Faculté des Sciences Gabriel, Université de Bourgogne, Dijon, France
}

Received May 25, 2011; Accepted August 22, 2011

DOI: $10.3892 / \mathrm{mmr} .2011 .682$

\begin{abstract}
Lung cancer is a malignant disease with poor outcome, which has led to a search for new therapeutics. The PI3K/Akt/ mTOR and Ras/raf/Erk pathways are key regulators of tumor growth and survival. In the present study, their roles were evaluated by MTT assay, flow cytometry and Western blotting in lung cancer cells. We found that a high efficacy of antitumor activity was shown with GDC-0941 treatment in two gefitinibresistant non-small cell lung cancer (NSCLC) cell lines, A549 and $\mathrm{H} 460$. In addition, $\mathrm{H} 460$ cells with activating mutations of PIK3CA were relatively more sensitive to GDC-0941 than A549 cells with wild-type PIK3CA. Furthermore, GDC-0941 was highly efficacious in combination with U0126 in inducing cell growth inhibition, $\mathrm{G}_{0}-\mathrm{G}_{1}$ arrest and cell apoptosis. These antitumor activities of combined treatment may be attributed to the alterations of $\mathrm{G}_{0}-\mathrm{G}_{1}$ phase regulators, apoptosis-related proteins and eukaryotic translation initiation factor 4B (eIF4B), induced by concomitant blockade of the PI3K/Akt/mTOR and Ras/raf/Erk pathways. In conclusion, this study suggests that multi-targeted intervention is the most effective treatment for tumors. Additionally, the blockade of PI3K, mTOR and Erk with GDC-0941 and MEK inhibitors shows promise for treating gefitinib-resistant NSCLC.
\end{abstract}

\section{Introduction}

Lung cancer is the leading cause of cancer mortality in the United States and worldwide. Non-small cell lung cancer (NSCLC) accounts for approximately $85 \%$ of lung cancer cases, and the majority of NSCLC patients do not benefit from chemotherapy, with a $16 \%$ overall 5 -year survival rate (1). The poor outcome for advanced lung cancer patients has led to a search for new therapeutics. Thus, the past decade has

Correspondence to: Dr Xiao-Hong Zhang, Medical School, Ningbo University, 818 Fenghua Road, Ningbo, Zhejiang 315211, P.R. China

E-mail: zhangxiaohong1@nbu.edu.cn

*Contributed equally

Key words: GDC-0941, PI3K, mTOR, Erk, synergize witnessed targeted therapy in NSCLC. However, gefitinib, a specific inhibitor of epidermal growth factor receptor (EGFR) tyrosine kinase, has shown efficacy only in a subgroup of NSCLC patients with EGFR mutations and overexpression. It was previously shown that EGFR-independent activation of the $\mathrm{PI} 3 \mathrm{~K} / \mathrm{Akt} / \mathrm{mammalian}$ target of rapamycin (mTOR) and Ras/ raf/Erk pathways may contribute to the unfavorable treatment outcome of gefitinib (2).

The PI3K/Akt/mTOR and Ras/raf/Erk signaling pathways play a key role in cell growth, proliferation and survival in a number of types of human cancer, and have received much attention as potential targets for the treatment of human cancer $(3,4)$. In human tumors, the PI3K/Akt/mTOR pathway is frequently constitutively activated through a variety of activating alterations. These activating alterations include loss, or inactivating mutations, of the tumor suppressor gene PTEN, which negatively regulates PI3K lipid kinase activity (5), the gain-of-function mutations in the PIK3CA gene itself encoding the p110a catalytic subunit of PI3K (6) and gene amplification of $A K T 1$ (7). mTOR, which integrates growth factor signaling together with nutrients and energy to regulate protein synthesis, cell growth and proliferation, has high activity in various types of cancer (8). Similarly, in the Ras/raf/Erk pathway, activating mutations in k-Ras and B-raf occur in more than $30 \%$ of all human tumors and in $60 \%$ of malignant melanomas, respectively (9). In addition, cancer cells depend on functional crosstalks and feedback mechanisms among key signaling pathways to drive cell growth and malignant transformation in unfavorable conditions. An example of these feedback loops is that inhibiting mTOR with rapamycin and its analogues leads to markedly activated Akt (10). Another example of these crosstalks among various molecular pathways is that inhibition of mTOR induces Ras/raf/Erk pathway activation through a PI3K-dependent feedback loop in human cancer (11). These findings reveal why first-generation targeted agents such as gefitinib are unlikely to prove totally effective in NSCLC treatment, and highlight the usefulness of multipoint interventions in NSCLC, leading us to assess the rationale for a combined inhibition of PI3K/Akt/mTOR and Ras/raf/Erk pathways in NSCLC cells.

GDC-0941 is a potent and selective oral dual inhibitor of class I PI3K and mTOR, and exhibits favorable pharmacokinetic and toxicological properties, which differentiate it 
from previously tested dual PI3K/mTOR inhibitors including PI-103, PI-540 and PI-620 (12). GDC-0941 demonstrates antitumor activity in a broad range of preclinical models including glioblastoma, ovarian and breast cancer xenografts, alone or in combination with other molecular-targeted agents $(13,14)$. However, the antitumor activity and the corresponding mechanism of GDC-0941, alone or in combination with MEK inhibitor U0126, in gefitinib-resistant NSCLC remains elusive.

In the present study, we selected two gefitinib-resistant NSCLC cell lines, A549 with a K-ras mutation and H460 with $K$-ras and PIK3CA mutations $(15,16)$. We examined the antitumor activity and its corresponding mechanisms of GDC-0941 alone or in combination with U0126 in A549 and H460 cells.

\section{Materials and methods}

Cells and culture. A549 and H460 were purchased from the cell bank of the Chinese Academy of Sciences. Cells were cultured in RPMI-1640 containing $10 \%$ fetal bovine serum at $37^{\circ} \mathrm{C}, 5 \% \mathrm{CO}_{2}$. Logarithmically growing cells were used in all experiments.

Chemicals and antibodies. Agents were obtained from the following suppliers: GDC-0941 from Merck (Darmstadt, Germany); U0126 (MEK inhibitor) and MTT from Sigma (St. Louis, MO, USA); coulter DNA prep reagents kit for cell cycle from Beckman Coulter (Fullerton, CA, USA). Antibodies were purchased from Cell Signaling Technology (Beverly, MA, USA), with the exception of the following antibodies: phospho-Akt ${ }^{\mathrm{Ser} 473}$, Akt, p70s6k and $\beta$-actin (Santa Cruz Biotechnology, Santa Cruz, CA, USA).

MTT assay. A549 and H460 cells were seeded at a density of 3,000 cells/well in 96-well plates, and after $24 \mathrm{~h}$ the cells were treated with various concentrations of drugs, either as a single agent or in combination. Following $72 \mathrm{~h}$ of treatment, $20 \mu \mathrm{l}$ of MTT [ $5 \mathrm{mg} / \mathrm{ml}$ in phosphate-buffered saline (PBS)] was added to each well and the plates were then incubated for $4 \mathrm{~h}$ at $37^{\circ} \mathrm{C}$. The medium was then placed in $200 \mu 1$ of DMSO. Absorbance was measured at $492 \mathrm{~nm}$ using a multiwell plate reader. Each experiment was performed in triplicate.

Flow cytometry. Cells were plated into 6-well plates and on the following day were treated with various concentrations of the drugs for $24 \mathrm{~h}$. Following the treatment, cells were fixed with ice-cold $70 \%$ ethanol. Subsequently, cells were incubated with cell cycle reagents from Beckman Coulter for $30 \mathrm{~min}$ at $4^{\circ} \mathrm{C}$ in the dark. Cell cycle distribution was then analyzed with FACSCalibur flow cytometer and CellQuest Pro software.

Western blotting. Cells were treated with various concentrations of drugs for $24 \mathrm{~h}$ and harvested with ice-cold NP-40 lysis buffer. Equal amounts of total protein were separated on polyacrylamide gels and then transferred onto PVDF membranes, which were incubated overnight with the primary antibodies. The following day, the membranes were incubated with the appropriate horseradish peroxidase-conjugated secondary antibodies. Proteins were detected by an ECL detection system.

\section{Results}

Synergistic inhibition of cell growth by a combination of GDC-0941 and U0126. As shown in Fig. 1A, GDC-0941 and U0126 induced a dose-dependent growth inhibition in A549 and H460 cells. In contrast to A549 cells, H460 cells were more sensitive to low-dose GDC-0941. In addition, H460 cells were also relatively more sensitive to U0126 compared to A549 cells. Notably, treatment of A549 and H460 cells with the combination of GDC-0941 and U0126 resulted in a more significant growth inhibition, as compared to treatment with the single drugs. Using the combination index (CI) isobologram analysis according to the method of Chou and Talalay, CI values for the combination of GDC-0941 and U0126 were found to be $<1$, indicating that U0126 $(5 \mu \mathrm{M})$ synergistically increased the antiproliferative effects of 0.05 and $0.1 \mu \mathrm{M}$ GDC-0941 in the two cell lines. Particularly in H460 cells, GDC-0941 $(0.1 \mu \mathrm{M})$ acted in robust synergy with U0126 $(5 \mu \mathrm{M})(\mathrm{CI}, 0.447)(\mathrm{CI}<1$ indicates synergy, whereas a $\mathrm{CI}>1$ indicates antagonism) (Fig. 1B).

Effects of GDC-0941 and U0126 on signaling pathways. To verify that the observed synergistic growth inhibitory effects of GDC-0941 in combination with U0126 are due to the inhibition of the PI3K/Akt/mTOR and Ras/raf/Erk pathways, we analyzed the activation status of downstream components by Western blotting. Treatment of A549 and H460 cells with GDC-0941 $(0.5 \mu \mathrm{M})$ for $24 \mathrm{~h}$ blocked phosphorylation of Akt. As expected, p-p70s6k was significantly impacted by GDC-0941 treatment, which changed in parallel with p-Akt in A549 and H460 cells. By contrast, phosphorylation of Erk was hardly impacted by GDC-0941. Similarly, Akt, p70s6k and Erk were not affected by GDC-0941. Meanwhile, treatment of H460 and A549 cells with U0126 $(5 \mu \mathrm{M})$ specifically and effectively inhibited phosphorylation of Erk, but not of Akt and p70s6k. The combination of GDC-0941 $(0.5 \mu \mathrm{M})$ and U0126 $(5 \mu \mathrm{M})$ simultaneously blocked phosphorylation of Akt, p70s6k and Erk in A549 and H460 cells. As expected, phosphorylated Akt was scarcely impacted by U0126, and phosphorylated Erk was also barely affected by GDC-0941 (Fig. 2).

GDC-0941 and U0126 synergistically induced cell cycle arrest. Based on the synergistic growth inhibitory response of A549 and H460 cells to the combination of GDC-0941 and U0126, we then examined their effects on cell cycle. Treatment of A549 and H460 cells with GDC-0941 $(0.5 \mu \mathrm{M})$ or U0126 $(5 \mu \mathrm{M})$ alone for $24 \mathrm{~h}$ induced a significant increase in $\mathrm{G}_{0}-\mathrm{G}_{1}$ DNA content. In addition, GDC-0941 $(0.5 \mu \mathrm{M})$ alone induced $\mathrm{G}_{0}-\mathrm{G}_{1}$ arrest more markedly in $\mathrm{H} 460$ than in A549 cells. Notably, a prominent action in blocking cell cycle progression in the two cells was observed and the majority of cells were arrested at $\mathrm{G}_{0}-\mathrm{G}_{1}$ when $\mathrm{A} 549$ and $\mathrm{H} 460$ cells were treated with GDC-0941 $(0.5 \mu \mathrm{M})$ and U0126 (5 $\mu \mathrm{M})$ (Fig. 3A and B).

Effects of GDC-0941 and U0126 on $G_{0^{-}} G_{1}$ cell cycle regulators, apoptosis-related proteins and the eukaryotic translation initiation factor. In A549 and H460 cells, treatment with GDC-0941 and U0126 alone for $24 \mathrm{~h}$ increased the expression of the cyclin-dependent kinase inhibitors p21, p27, and tumor suppressor protein $\mathrm{p} 53$, which are associated with $\mathrm{G}_{0}-\mathrm{G}_{1}$ 

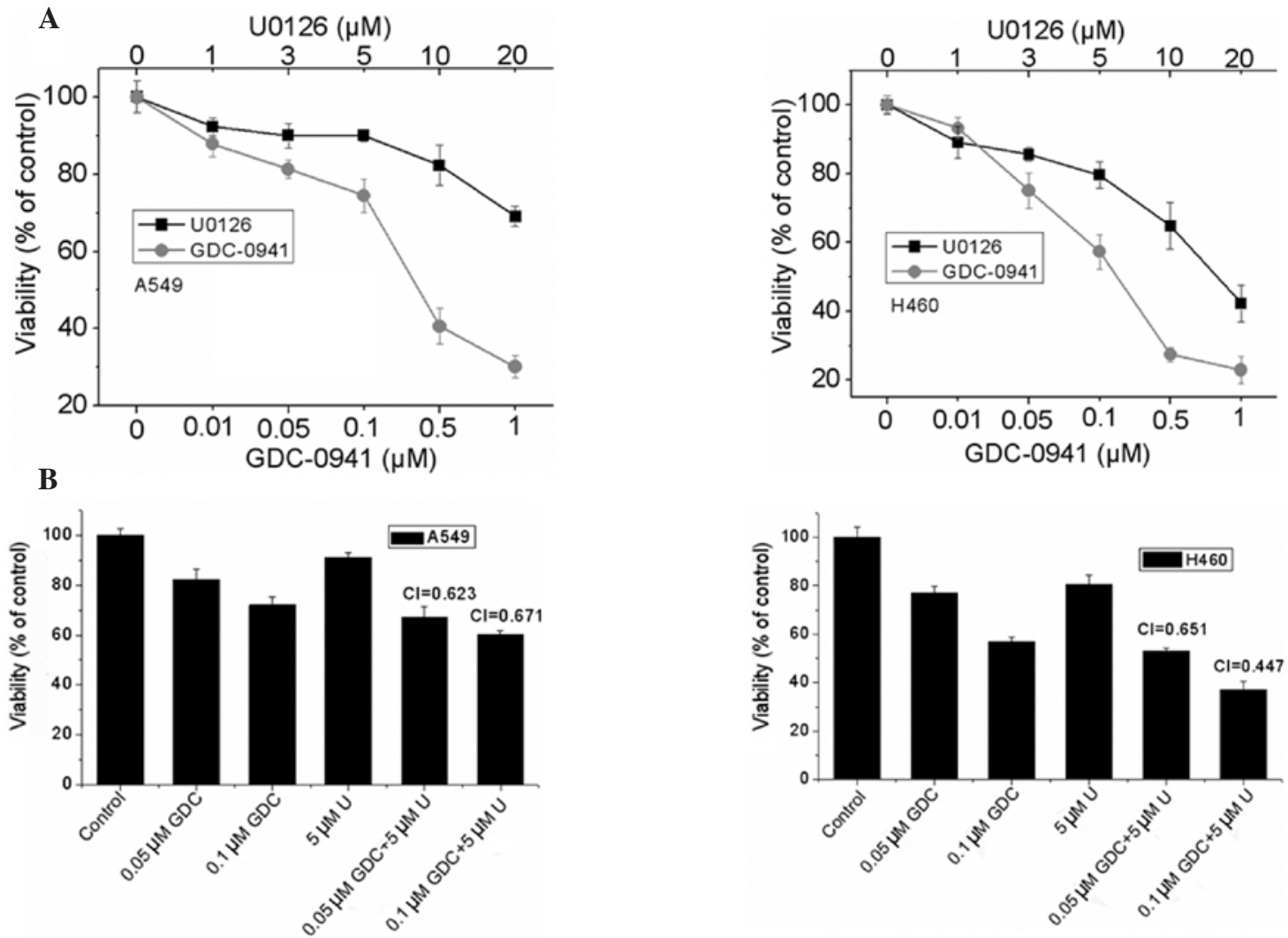

Figure 1. Growth inhibitory effects of GDC-0941 and U0126 on human NSCLC cell lines A549 and H460. (A) A549 and H460 cells were seeded in 96-well plates and treated with the indicated concentrations of GDC-0941 and U0126. Following 72 h of treatment, cell viability was determined using a MTT assay. The cell growth data are the means \pm SD of three independent experiments. (B) Combination analysis was performed using the method described by Chou and Talalay. CI, combination index.

arrest. As expected, co-treatment of A549 and H460 cells with GDC-0941 and U0126 markedly increased the expression of these inhibitors. Similarly, incubation of A549 and H460 cells with GDC-0941 or U0126 alone led to a decreased expression of cell cycle protein cyclin D1, cyclin E1 and phosphorylation of the eukaryotic translation initiation factor eIF4B. The combination of GDC-0941 and U0126 significantly downregulated their levels. In addition, GDC-0941 up-regulated the expression of the apoptosis protein Bad in the two cells, and U0126 only induced its expression in A549 but not in H460 cells. The combination of GDC-0941 and U0126 markedly induced the expression of $\mathrm{Bad}$ and down-regulated the expression of anti-apoptosis protein bcl-2 in the two cells (Fig. 4).

\section{Discussion}

Receptor tyrosine kinases are overexpressed with activating mutations in NSCLC or other solid tumors, thus protein kinases have gained much attention as drug targets in cancer therapy (17). This strategy has led to the development of HER2-targeted herceptin, which is mostly used to treat HER2-overexpression in breast cancer (18); and EGFRtargeted gefitinib/erlotinib, which is mainly used to treat NSCLC or SCLC. However, gefitinib and erlotinib produced only a $12-27 \%$ objective response rate in advanced NSCLC patients (19). Although EGFR mutations are a crucial determinant of objective responses to gefitinib and erlotinib, the activating status of downstream kinase signaling pathways

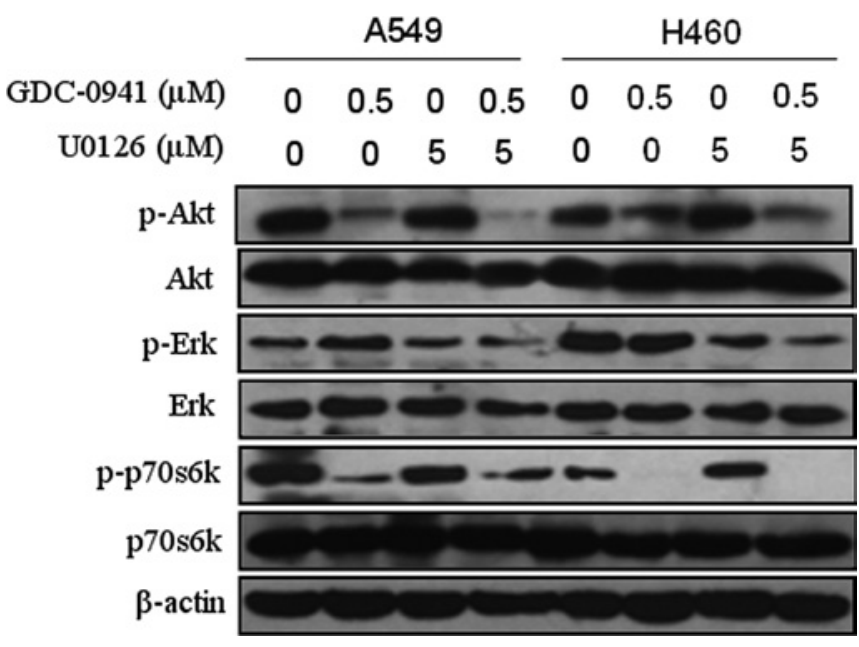

Figure 2. Effect of GDC-0941 and U0126 on the downstream components of PI3K and Ras in A549 and H460 cells. Cells were treated with GDC-0941 and U0126 alone or in combination at the doses shown for $24 \mathrm{~h}$. Cell lysates were subjected to immunoblot analysis with the indicated antibodies.

such as PI3K/Akt/mTOR and Ras/raf/Erk is closely associated with gefitinib/erlotinib sensitivity of NSCLC patients in clinical studies (20). Therefore, in the past decades, the overemphasis on protein kinases has shadowed lipid kinases, such as PI3K and mTOR, which are implicated in carcinogenesis, inflammation and autoimmune conditions. However, the 
A

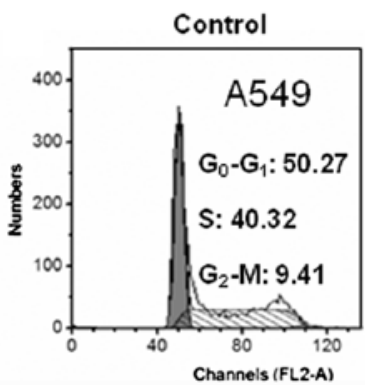

B

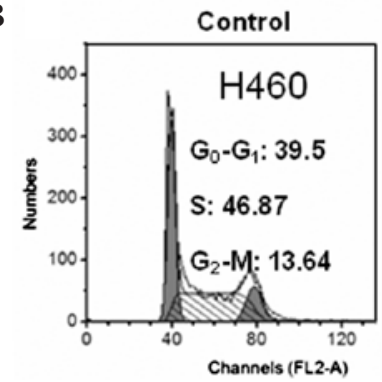

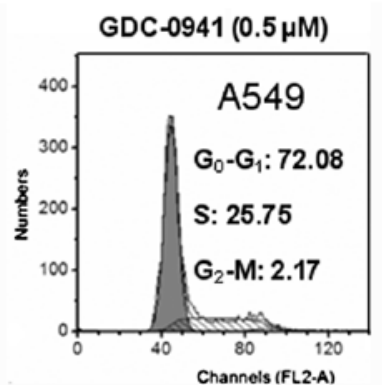

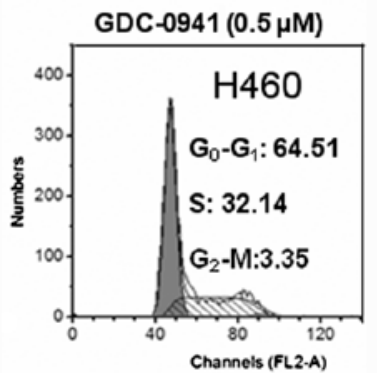

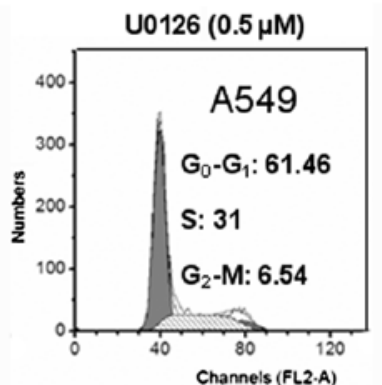
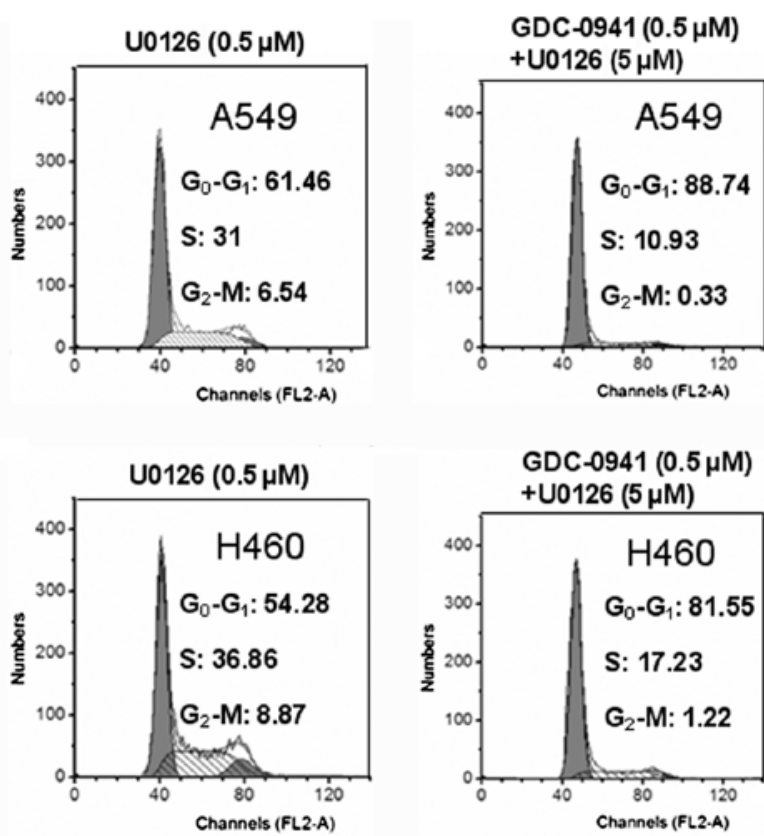

GDC-0941 (0.5 $\mu \mathrm{M})$

$+\mathrm{U} 0126(5 \mu \mathrm{M})$

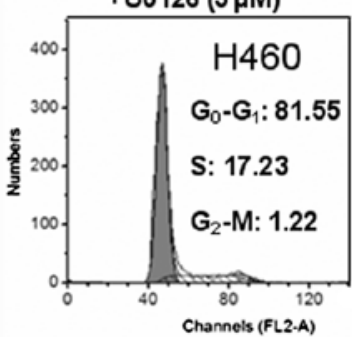

Figure 3. Effect of GDC-0941 and U0126, either alone or in combination, on cell cycle progression in A549 and H460 cells. (A) A549 cells and (B) H460 cells were treated with GDC-0941 or U0126 alone, or in combination, for $24 \mathrm{~h}$. Cells were collected and stained with cell cycle agents as detailed in Materials and methods. Flow cytometric analysis was then used for cell cycle distribution.

constitutive PI3K pathway has triggered the development of a series of PI3K/mTOR dual inhibitors such as NVP-BEZ235 (21), ZSTK474 (22) and GDC-0941 (12) since the first PI3K/ mTOR dual inhibitor PI-103 was developed in 2006 (23). These molecules inhibit PI3K and mTOR through the same mechanism as PI-103, but with different pharmacokinetic and solubility properties. GDC-0941 as a single agent was efficacious in HER2-positive breast cancer cells, and the combination of GDC-0941 with the HER2 inhibitor trastuzumab resulted in synergistic growth inhibition (14). Therefore, GDC-0941 alone or in combination with MEK inhibitors may provide a strong rationale for the treatment of gefitinib-resistant NSCLC.

In the present study, $\mathrm{H} 460$ cells were more sensitive to GDC-0941 compared to A549 cells, due to H460 cells with activating mutations of the PIK3CA gene (24). In general, tumor cells depend on the constitutive activation of key signaling pathways for growth, proliferation and survival, termed oncogene addiction (25). Thus, such tumor cells are more sensitive to specific inhibitors of these signaling pathways. The mechanism by which GDC-0941 induced growth suppression is by the simultaneous blockade of PI3K and mTOR kinase activity. However, a series of previous studies has shown that the single targeted inhibitors of mTOR, including CCI-779, RAD001 and AP23573 markedly activate Akt by inhibiting mTOR, which may help to explain the fact that rapamycin analogues have not shown good antitumor activity in clinical trials (10). Therefore, these preclinical and clinical data support that PI3K/mTOR dual inhibitors blocking the Akt activation induced by mTOR inhibition may have a greater therapeutic efficacy in the clinic. Additionally, the PI3K/Akt/mTOR and Ras/raf/Erk signaling pathways are simultaneously inhibited by the combined treatment of GDC-0941 and U0126, which induced synergistic growth inhibition in A549 and H460 cells. It is plausible that concomitant blockade of the PI3K/mTOR and MEK kinases

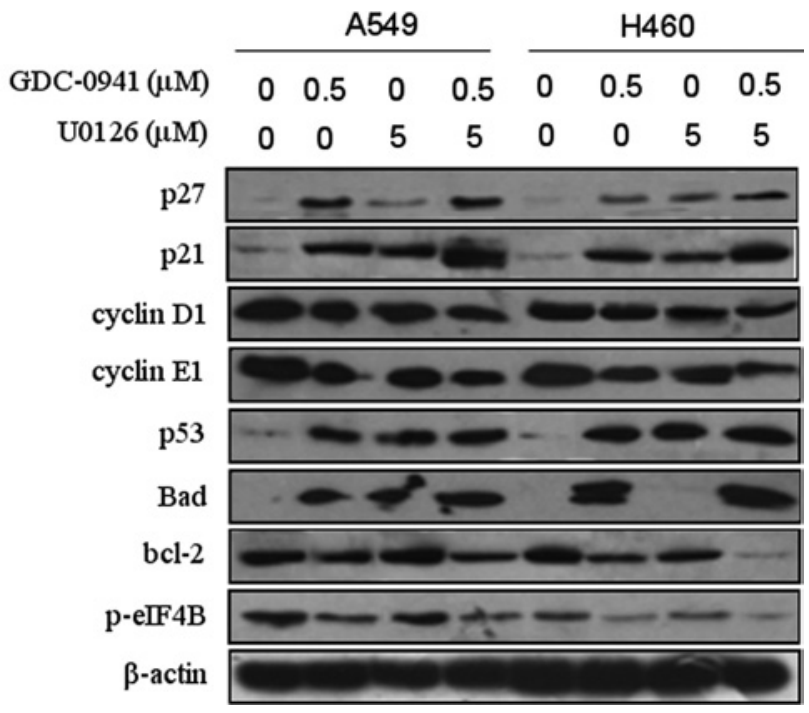

Figure 4. Effect of GDC-0941 and U0126 on cell cycle regulatory proteins, apoptosis-related proteins and eukaryotic translation initiation factor eIF4B in A549 and H460 cells. Cells were treated with GDC-0941 alone or in combination with U0126 at the doses shown for $24 \mathrm{~h}$ as described in Materials and methods. Membranes were probed with anti-p27, p21, cyclin D1, cyclin E1, p53, Bad, bcl-2, p-eIF4B and $\beta$-actin antibodies, and detected by an ECL detection system.

activities has a synergistic effect. Although our data have not indicated that the crosstalk exists between the PI3K/Akt $/ \mathrm{mTOR}$ and Ras/raf/Erk signaling pathways, we found that p70s6k phosphorylation was partially inhibited by high-dose U0126 $(40 \mu \mathrm{M})$ in the two cells, supporting the fact that mTOR acts downstream of Erk (26). Additionally, a recent study showed that inhibition of mTORC1 leads to the activation of the Ras/raf/Erk pathway through a PI3K-dependent feedback loop 
in human cancer (11), suggesting that crosstalk mechanisms exist widely in signaling networks. In this context, it has been shown that $\mathrm{G}_{0}-\mathrm{G}_{1}$ phase arrest was synergistically induced in the two cells treated with a combination of GDC-0941 and U0126, further underscoring the potential of a combined therapeutic strategy with the PI3K/Akt/mTOR and Ras/raf/Erk signaling pathway inhibitors for the treatment of human cancer.

Proliferation of tumor cells is regulated by cell cycle-dependent cyclins, cyclin dependent kinases (CDKs) and CDK inhibitors (CDKIs) (27). Cyclin D isoforms bind to CDK4 and CDK6, which aid in the progression through the early $\mathrm{G}_{1}$ phase of the cell cycle. Cyclin E/CDK2 complexes play a key role in the transition from the $G_{1}$ to $S$ phase of the cell cycle. In addition, the activity of CDKs is regulated by cyclins and inhibited by $\mathrm{CDK}$ inhibitors such as p21 and p27. In tumor cells, loss or low expression of another established tumor suppressor p53, which regulates p21 expression, may lead to loss of cell cycle checkpoints, deregulated apoptosis and increased genetic instability (28). In the present study, our results showed that GDC or U0126 exerts its effects on $G_{0}-G_{1}$ arrest via the up-regulation of p21, p53 and p27, and the simultaneous down-regulation of cyclin D1 and cyclin E1. The synergistically anti-proliferative effects of GDC-0941 in combination with U0126 were accounted for by marked increases in the expression of p21, p53 and p27 proteins, and significant reductions in cyclin D1 and cyclin E1 (Fig. 4). GDC-0941 was found to show high efficacy and its antitumor activity was markedly synergized with U0126 in A549 and H460 cells, which was associated with a strong $\mathrm{G}_{0}-\mathrm{G}_{1}$ arrest in cell cycle progression and cell apoptosis. Additionally, GDC-0941 not only induced cell cycle arrest but also induced cell apoptosis, whereas PI-103 only induced cytostasis. We showed that the tumor suppression of the combination of GDC-0941 and U0126 was also associated with the synergistically up-regulating apoptotic protein Bad and the down-regulating anti-apoptosis protein bcl-2. Data from recent studies suggest that the antitumor activity mediated by GDC-0941 and U0126 is linked to a sequential regulation of cell cycle proteins and apoptosis proteins, which clearly play a significant role in tumor growth and survival.

eIF4B increases the eukaryotic translation initiation factor 4F (eIF4F) activity by increasing the eukaryotic translation initiation factor 4A (eIF4A) RNA helicase activity, and interacts with the eukaryotic translation initiation factor 3 (eIF3) (29). eIF4A-mediated helicase activity and the interaction between eIF4B and eIF3 are enhanced by eIF4B phosphorylation, which finally potentiates the cap-dependent translation. The PI3K/Akt/mTOR and Ras/raf/Erk pathways were found to converge on eIF4B to regulate its phosphorylation and activity (30). In the present study, the results showed that phosphorylation of eIF4B on Ser 422 was concomitantly inhibited by GDC-0941 and U0126, which may cooperatively inhibit cap-dependent translation initiation in the two cell lines. These data clarify the reason for the co-blockade of the $\mathrm{PI} 3 \mathrm{~K} / \mathrm{Akt} / \mathrm{mTOR}$ and Ras/raf/Erk pathways exhibiting markedly increased antitumor activity.

For the majority of types of cancer, there are a number of factors promoting tumor growth, proliferation and survival. Widely intricate feedback regulation and crosstalk in signaling networks also exist, as shown for mTOR inhibition-mediated Akt activation (10). This is one of the main reasons for the relative failure of the first generation single-targeted inhibitors such as gefitinib and erlotinib. Therefore, preclinical studies highlighted that combined treatment with targeted inhibitors that concurrently block multiple signaling pathways produced synergistic antitumor activity $(31,32)$. Our data showed that synergistic growth inhibitory effects of GDC-0941 in combination with U0126 were observed in A549 and H460 cells, further verifying the importance of multi-targeted therapeutics. Although there are insufficient clinical data to determine whether PI3K/mTOR dual inhibitors are powerful therapeutic agents alone or are more effective in combination with other targeted inhibitors, it is conceivable that the concomitant inhibition of PI3K, mTOR and Erk using GDC-0941 and MEK inhibitors provides a promising potential for treating gefitinibresistant NSCLC.

\section{Acknowledgements}

This study was partly sponsored by the K.C.Wong Magna Fund in Ningbo University and supported by the National Science Foundation of China (Grant No. 30972466 and 81102121). We are also grateful to Chen-Wei Xu for cell culture, and Shi-Yong Chen for useful discussion.

\section{References}

1. Paez JG, Jänne PA, Lee JC, et al: EGFR mutations in lung cancer: correlation with clinical response to gefitinib therapy. Science 304: 1497-1500, 2004.

2. Cappuzzo F, Magrini E, Ceresoli GL, et al: Akt phosphorylation and gefitinib efficacy in patients with advanced non-small-cell lung cancer. J Natl Cancer Inst 96: 1133-1141, 2004.

3. Cheng JQ, Lindsley CW, Cheng GZ, Yang H and Nicosia SV: The Akt/PKB pathway: molecular target for cancer drug discovery. Oncogene 24: 7482-7492, 2005.

4. Downward J: Targeting RAS signalling pathways in cancer therapy. Nat Rev Cancer 3: 11-22, 2003.

5. Alimonti A, Carracedo A, Clohessy JG, et al: Subtle variations in Pten dose determine cancer susceptibility. Nat Genet 42: 454-458, 2010.

6. Samuels Y, Wang Z, Bardelli A, et al: High frequency of mutations of the PIK3CA gene in human cancers. Science 304: 554, 2004

7. Liu LZ, Zhou XD, Qian G, Shi X, Fang J and Jiang BH: AKT1 amplification regulates cisplatin resistance in human lung cancer cells through the mammalian target of rapamycin/p70S6K1 pathway. Cancer Res 67: 6325-6332, 2007.

8. Bjornsti MA and Houghton PJ: The TOR pathway: a target for cancer therapy. Nat Rev Cancer 4: 335-348, 2004.

9. Brose MS, Volpe P, Feldman M, et al: BRAF and RAS mutations in human lung cancer and melanoma. Cancer Res 62: 6997-7000, 2002.

10. O'Reilly KE, Rojo F, She QB, et al: mTOR inhibition induces upstream receptor tyrosine kinase signaling and activates Akt. Cancer Res 66: 1500-1508, 2006.

11. Carracedo A, Ma L, Teruya-Feldstein J, et al: Inhibition of mTORC1 leads to MAPK pathway activation through a PI3Kdependent feedback loop in human cancer. J Clin Invest 118 : 3065-3074, 2008.

12. Folkes AJ, Ahmadi K, Alderton WK, et al: The identification of 2(1 H-Indazol-4-yl)-6-(4-methanesulfonyl-piperazin-1-ylmethyl)4-morpholin-4-yl-thieno [3, 2-d] pyrimidine (GDC-0941) as a potent, selective, orally bioavailable inhibitor of class I PI3 kinase for the treatment of cancer. J Med Chem 51: 5522-5532, 2008.

13. Raynaud FI, Eccles SA, Patel S, et al: Biological properties of potent inhibitors of class I phosphatidylinositide 3-kinases: from PI-103 through PI-540, PI-620 to the oral agent GDC-0941. Mol Cancer Ther 8: 1725-1738, 2009.

14. Junttila TT, Akita RW, Parsons K, et al: Ligand-independent HER2/HER3/PI3K complex is disrupted by trastuzumab and is effectively inhibited by the PI3K inhibitor GDC-0941. Cancer Cell 15: 429-440, 2009. 
15. Janmaat ML, Rodriguez JA, Gallegos-Ruiz M, Kruyt FA and Giaccone G: Enhanced cytotoxicity induced by gefitinib and specific inhibitors of the Ras or phosphatidyl inositol-3 kinase pathways in non-small cell lung cancer cells. Int J Cancer 118: 209-214, 2006.

16. Whyte DB and Holbeck SL: Correlation of PIK3Ca mutations with gene expression and drug sensitivity in NCI-60 cell lines. Biochem Biophy Res Commun 340: 469-475, 2006.

17. Zwick E, Bange J and Ullrich A: Receptor tyrosine kinases as targets for anticancer drugs. Trends Mol Med 8: 17-23, 2002.

18. Tran T, Orlova A, Igor SIVAEV, Sandström M and Tolmachev V: Comparison of benzoate-and dodecaborate-based linkers for attachment of radioiodine to HER2-targeting Affibody ligand. Int J Mol Med 19: 485-493, 2007.

19. Italiano A, Vandenbos FB, Otto J, et al: Comparison of the epidermal growth factor receptor gene and protein in primary non-small-cell-lung cancer and metastatic sites: implications for treatment with EGFR-inhibitors. Ann Oncol 17: 981-985, 2006.

20. McCubrey JA, Steelman LS, Abrams SL, et al: Roles of the RAF/ MEK/ERK and PI3K/PTEN/AKT pathways in malignant transformation and drug resistance. Adv Enzyme Regul 46: 249-279, 2006.

21. Maira SM, Stauffer F, Brueggen J, et al: Identification and characterization of NVP-BEZ235, a new orally available dual phosphatidylinositol 3-kinase/mammalian target of rapamycin inhibitor with potent in vivo antitumor activity. Mol Cancer Ther 7: 1851-1863, 2008.

22. Yaguchi S, Fukui Y, Koshimizu I, et al: Antitumor activity of ZSTK474, a new phosphatidylinositol 3-kinase inhibitor. J Natl Cancer Inst 98: 545-556, 2006.

23. Fan QW, Knight ZA, Goldenberg DD, et al: A dual PI3 kinase/ mTOR inhibitor reveals emergent efficacy in glioma. Cancer Cell 9: 341-349, 2006
24. Vasudevan KM, Barbie DA, Davies MA, et al: AKT-independent signaling downstream of oncogenic PIK3CA mutations in human cancer. Cancer Cell 16: 21-32, 2009.

25. Weinstein IB ans Joe A: Oncogene addiction. Cancer Res 68: 3077-3080, 2008.

26. Ma L, Chen Z, Erdjument-Bromage H, Tempst P and Pandolfi PP: Phosphorylation and functional inactivation of TSC2 by Erk implications for tuberous sclerosis and cancer pathogenesis. Cell 121: 179-193, 2005.

27. Grana $X$ and Reddy EP: Cell cycle control in mammalian cells: role of cyclins, cyclin dependent kinases (CDKs), growth suppressor genes and cyclin-dependent kinase inhibitors (CKIs). Oncogene 11: 211-219, 1995.

28. Levine AJ: p53, the cellular gatekeeper for growth and division. Cell 88: 323-331, 1997.

29. Rozen F, Edery I, Meerovitch K, Dever TE, Merrick WC and Sonenberg N: Bidirectional RNA helicase activity of eucaryotic translation initiation factors $4 \mathrm{~A}$ and $4 \mathrm{~F}$. Mol Cell Biol 10: 1134-1144, 1990

30. Shahbazian D, Roux PP, Mieulet V, et al: The mTOR/PI3K and MAPK pathways converge on eIF4B to control its phosphorylation and activity. Embo J 25: 2781-2791, 2006.

31. Engelman JA, Chen L, Tan X, et al: Effective use of PI3K and MEK inhibitors to treat mutant Kras G12D and PIK3CA H1047R murine lung cancers. Nat Med 14: 1351-1356, 2008.

32. Legrier ME, Yang CPH, Yan HG, et al: Targeting protein translation in human non-small cell lung cancer via combined MEK and mammalian target of rapamycin suppression. Cancer Res 67: 11300-11308, 2007. 